\title{
Dressing Transformations and Poisson Group Actions
}

By

\author{
MichaeL。A. Semenov-Tian-ShanskY*
}

\begin{abstract}
Poisson properties of dressing transformations in soliton theory are explained.
\end{abstract}

Transformation groups for soliton equations were defined in [1]. They were also implicit in the earlier paper [2] and are known under an alternative name of dressing transformation groups (which is the one we shall use in the sequel). For a modern and comprehensible treatment see $[3,4]$. These groups are generally regarded as the hidden symmetry groups for integrable systems. The infinitesimal action of these groups was also studied by direct methods (see [5] for a review).

We now come to a major puzzle that remained open in the theory. Given the fundamental role of Poisson brackets on the phase space one would expect that dressing transformation group preserves them. Surprisingly, however, this fails to be so in general*). It is particularly interesting to understand this phenomenon in order to decide whether the dressing transformation group survives quantization. Normally this would have been the case if it preserved the Poisson brackets. Since it fails to do so, the situation becomes obscure.

The aim of the present paper is to explain the Poisson properties of dressing transformations. The general set-up for the answer partly results from my conversations with V. Drinfel'd which I gratefully acknowledge. It is important to observe that dressing transformation

\footnotetext{
Communicated by M. Sato, May 28, 1985.

* Leningrad Branch of the Steklov Mathematical Institute, 191011, Leningrad USSR.

*) This was observed e.g. in [6] and also by the author (unpublished).
} 
groups (which in typical cases are related to Kac-Moody Lie groups, or loop groups) carry a natural Poisson structure which is uniquely defined by the specific type of the Riemann-Hilbert problem entering the definition of dressing transformations. (A connection between Poisson structures and Riemann problems is central in the so called r-matrix formalism, see [7].) This Poisson structure has an important property explicitly stated for the first time in [8]. (Although taking its origin in earlier papers, cf. [7], [10].)

Definition [8]. A Lie group $G$ is called a Poisson Lie group*) if a Poisson bracket is fixed on $G$ such that multiplication $G \times G \rightarrow G$ is a Poisson mapping, the space $G \times G$ being equipped with the product Poisson structure.

Let $M$ be a Poisson manifold. An action $G \times M \rightarrow M$ is called Poisson action if it is a Poisson mapping, the space $G \times M$ being equipped with the product Poisson structure.

We refer the reader to [9] for the definition and standard properties of Poisson manifolds. In the sequel we shall make an extensive use of various concepts from this paper.

Clearly, a Poisson action whenever non-trivial does not preserve Poisson brackets on $M$ (cf. formulae (15), (22) below). However, the resulting category is quite rich in geometric structures (e.g. for Poisson $G$-spaces there are analogs of the Hamiltonian reduction technique, cf. $\mathrm{n}^{\circ} 3$ below).

Our main result may be now stated as follows. Dressing transformation groups define a Poisson group action.

In order to put it more precily, we must have a closer look at the definition of dressing transformations. Usually they are regarded as acting not on the phase space $B$ itself whose points are represented by Lax operators, but rather on the fiber bundle $P$ over $B$ consisting of wave function. Ordinary definition used in [1], [3], [4] does not lead to an action on $B$ since dressing transformations do not preserve the normalization of wave functions (cf. formula (45) below). However, there is a clever way around this obstacle: one can combine dressing and gauge transformations in a natural way so as to get a group action on the phase space itself. This use of gauge freedom is in

*) I prefer this term to the one originally used in [8] which was 'Hamilton Lie groups'. 
fact crucial since it changes the composition law of dressing transformations. With the group action on the phase space at hand, a question on its Poisson properties may be correctly posed and solved. In passing we discover a new Poisson structure which is in a sence dual to the one studied in [7], [8]. It is precisely this new Poisson structure that is relevant for the Poisson properties of dressing transformations. It also plays an important role in the description of Lax equations (as explained in sections 3-5 below). For technical reasons we shall mainly deal with the difference Lax equations. The results for the continuous case are analogous and are stated at the end of Section 6.

Our result has a clear bearing on the quantization problem. Namely, it indicates that the dressing transformation group itself should be quantized, its matrix elements becoming $q$-numbers rather than ordinary c-numbers. An appropriate algebraic notion of a "quantum group" was recently proposed by V. Drinfel'd. I hope to consider these questions more closely in a separate publication.

\section{Acknowledgements}

The author wishes to thank V. Drinfel'd for very valuable and stimulating discussions. Discussions held during the International Taniguchi Symposium at Kyoto and Katata in August, 1984 were also of considerable support to me. I am deeply grateful to the organizers of Taniguchi Symposium for their hospitality. The present paper gives an answer to the question enrolled in the list of open problems published by the Taniguchi Foundation.

\section{§ 1. Basic Algebra Related to the Yang-Baxter Identity}

The technical background for our study in provided by the $\mathrm{r}$ matrix formalism as developed in [7], [8]. We begin with a brief review of the relevant facts.

Definition 1. Let $g$ be a Lie algebra, $R \in$ End $g$ a linear operator. We say $(\mathfrak{g}, R)$ is a Baxter Lie algebra if

(i) There is a nondegenerate invariant scalar product on $\mathfrak{g}$

(ii) $R$ is skew-symmetric and satisfies the Yang-Baxter identity 
(1) $[R X, R Y]=R([R X, Y]+[X, R Y])-[X, Y], X, Y \in \mathfrak{g}$ 。

An operator $R$ satisfying the conditions above is called a classical r-matrix. We refer the reader to [7] for a discussion of how the present definition links with tensor formalism used in [10].

\section{Proposition 1. (i) Let}

$$
[X, Y]_{R}=\frac{1}{2}([R X, Y]+[X, R Y]) \text {. }
$$

Then (1) implies that (2) is a Lie bracket. We denote by $\mathfrak{g}_{R}$ the corresponding Lie algebra (with the same underlying linear space). (ii) Let $R_{ \pm}=\frac{1}{2}(R \pm 1)$. Then $R_{ \pm}: \mathfrak{g}_{R} \rightarrow \mathfrak{g}$ are Lie algebra homomorphisms。

Let $\mathfrak{d}=\mathfrak{g} \oplus \mathfrak{g}$. Let ${ }^{\delta} \mathrm{g} \subset \mathfrak{d}$ be its diagonal subalgebra. We embed $\mathfrak{g}_{R} \subseteq \mathfrak{d}$ via $X \mapsto\left(R_{+} X, R_{-} X\right)$. Note that $R_{+}-R_{-}=1$ and hence every $X \in \mathfrak{g}$ admits a unique decomposition

$$
X=X_{+}-X_{-}
$$

with $\left(X_{+}, X_{-}\right) \in \mathfrak{g}_{R} \subset \delta$. Equip $\delta$ with the inner product

$$
\left\langle\left(X_{1}, Y_{1}\right),\left(X_{2}, Y_{2}\right)\right\rangle=\left(X_{1}, X_{2}\right)-\left(Y_{1}, Y_{2}\right), \quad X_{i}, Y_{i} \in \mathfrak{g} .
$$

Proposition 2. (i) $\delta={ }^{\delta} \mathfrak{g}+g_{R}$ as a linear space. (ii) Let $P_{\delta_{\mathfrak{g}}}, P_{\mathfrak{g}_{R}}$, be projection operators onto ${ }^{\delta} \mathrm{g}, \mathfrak{g}_{R}$ parallel to the complementary subalgebra. Then

$$
R_{\mathrm{b}}=P_{\delta_{\mathrm{g}}}-P_{\mathrm{g}_{R}} \in \text { End } \mathfrak{D}
$$

satisfies the identity (1) and is skew-symmetric with respect to (4).

By virtue of Proposition 2, $\left(\delta, R_{\triangleright}\right)$ is again a Baxter Lie algebra. We shall call $\left(\mathfrak{D}, R_{\mathfrak{b}}\right)$ the square of $(\mathfrak{g}, R)$.

Note. In a more general way, one can define the square of an arbitrary Lie bialgebra. This general definition is due to V. Drinfel'd ([8]).

We now turn to the corresponding notions for Lie groups. Let $G, G_{R}$ be local Lie groups corresponding to $\mathfrak{g}, \mathfrak{g}_{R}$. There are natural 
homomorphisms $R_{ \pm}: G_{R} \rightarrow G: x \mapsto x_{ \pm}$which correspond to the Lie algebra homomorphisms denoted by the same letters. Let $D=G \times G$. We embed $G_{R} \subset D$ via $x \mapsto\left(x_{+}, x_{-}\right)$. Each $x \in G$ admits a unique decomposition

$$
x=x_{+} x_{-}^{-1}
$$

with $\left(x_{+}, x_{-}\right) \in G_{R} \subset D$. This mapping establishes a local homeomorphism of $G_{R}$ into $G$. The factorization problem (6) may be regarded as an abstract version of the Riemann problem.

Let ${ }^{\delta} G \subset G \times G$ be the diagonal subgroup. For each $(x, y) \in D$ there exists a unique factorization

$$
(x, y)=(\xi, \xi)\left(h_{+}, h_{-}\right), \quad(\xi, \xi) \in{ }^{\delta} G, \quad\left(h_{+}, h_{-}\right) \in G_{R^{*}}
$$

\$2。

Classical $r$-matrices are used to define Poisson structures on Lie groups. We shall eventually need quite a lot of them, their mutial relations being of importance to describe integrable systems, their dynamics and their transformation groups. A fairly general class of Poisson brackets is defined as follows.

Let $H$ be a Lie group, $\mathfrak{h}$ its Lie algebra. Suppose, there is an invariant scalar product on $\mathfrak{h}$. For $\varphi \in C^{\infty}(H)$ let us denote by $\nabla_{\varphi}, \nabla_{\varphi}^{\prime} \in \mathfrak{h}$ its left and right gradients defined by

$$
\begin{aligned}
\left(\nabla_{\varphi}(x), \xi\right) & =\left(\frac{d}{d t}\right)_{t=0} \varphi\left(e^{t \xi} x\right), \\
\left(\nabla_{\varphi}^{\prime}(x), \xi\right) & =\left(\frac{d}{d t}\right)_{t=0} \varphi\left(x e^{t \xi}\right), \quad \xi \in \mathfrak{h} .
\end{aligned}
$$

Put

(9) $\{\varphi, \phi\}_{\left(R, R^{\prime}\right)}=\frac{1}{2}\left(R^{\prime}\left(\nabla_{\varphi}^{\prime}\right), \nabla_{\psi}^{\prime}\right)+\frac{1}{2}\left(R\left(\nabla_{\varphi}\right), \nabla_{\psi}\right), R, R^{\prime} \in$ End $\mathfrak{h}$.

Theorem 1. Suppose $R, R^{\prime} \in$ End $\mathfrak{h}$ are skew-symmetric and satisfy the Yang-Baxter identity (1). Then (9) is a Poisson bracket on $H$.

Sketch of a proof. (i) Consider first the right and left brackets (cf. [11]).

$$
\{\varphi, \phi\}_{r}=\left(R^{\prime}\left(\nabla_{\varphi}^{\prime}\right), \nabla_{\psi}^{\prime}\right), \quad\{\varphi, \phi\}_{e}=\left(R\left(\nabla_{\varphi}\right), \nabla_{\psi}\right) .
$$

Denote by $\eta_{r}, \eta_{e}$ the corresponding Hamiltonian operators. By definition, 
the Schouten bracket of Hamiltonian operators is the right hand side of the Jacobi identity. One checks, by direct computation, that

$$
\begin{aligned}
& {\left[\eta_{e}, \eta_{e}\right]\left(d \varphi_{1}, d \varphi_{2}, d \varphi_{3}\right)=\left(\nabla_{\varphi_{1}},\left[R\left(\nabla_{\varphi_{2}}\right), R\left(\nabla_{\varphi_{3}}\right)\right]\right)+\text { c. p. }} \\
& {\left[\eta_{r}, \eta_{r}\right]\left(d \varphi_{1}, d \varphi_{2}, d \varphi_{3}\right)=-\left(\nabla_{\varphi_{1}}^{\prime},\left[R\left(\nabla_{\varphi_{2}}^{\prime}\right), R\left(\nabla_{\varphi_{3}}^{\prime}\right)\right]\right)-\text { c. p. }}
\end{aligned}
$$

For obvious reasons one has also $\left[\eta_{e}, \eta_{r}\right]=0$ (since right and left shifts commute). Now, the r-matrices $R, R^{\prime}$ satisfy the classical YangBaxter identity (1). Hence (11) simplifies to

$$
\left[\eta_{e}, \eta_{e}\right]=-\left[\eta_{r}, \eta_{r}\right]=\left(\nabla_{\varphi_{1}},\left[\nabla_{\varphi_{2}}, \nabla_{\varphi_{3}}\right]\right) \text {. }
$$

It follows that $\eta_{e} \pm \eta_{r}$ has zero Schouten bracket with itself.

We denote by $H_{\left(R, R^{\prime}\right)}$ the group $H$ equipped with the Poisson bracket (9).

Theorem 2. Multiplication $H \times H \rightarrow H$ induces Poisson mappings

$$
\begin{aligned}
& H_{(R,-R)} \times H_{\left(R, R^{\prime}\right)} \rightarrow H_{\left(R, R^{\prime}\right)}, \\
& H_{\left(R, R^{\prime}\right)} \times H_{\left(R^{\prime},-R^{\prime}\right)} \rightarrow H_{\left(R, R^{\prime}\right)} .
\end{aligned}
$$

Proof. Let $\rho_{x}, \lambda_{x}$ be the right and left translation operators by an element $x \in H$ acting on $C^{\infty}(H)$. By definition, (13) is a Poisson mapping if

$$
\{\varphi, \phi\}_{\left(R, R^{\prime}\right)}(x y)=\left\{\rho_{y} \varphi, \rho_{y} \psi\right\}_{(R,-R)}(x)+\left\{\lambda_{x} \varphi, \lambda_{x} \psi\right\}_{\left(R, R^{\prime}\right)}(y) .
$$

Let $\eta_{R, R^{\prime}}^{\prime}$ be the Hamiltonian operator associated with (9) in the left invariant frame. Obviously, one has

$$
2 \eta_{R, R^{\prime}}^{\prime}(x)=R^{\prime}+\operatorname{Ad} x^{-1} \circ R \circ \operatorname{Ad} x
$$

whence

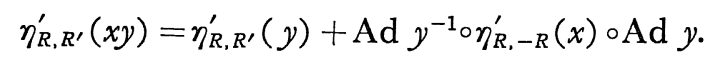

From the definition (8) of right gradients we get

$$
\nabla_{\lambda_{x} \varphi}^{\prime}(y)=\nabla_{\varphi}^{\prime}(x y), \quad \nabla_{\rho_{y} \varphi}^{\prime}(x)=\operatorname{Ad} y \cdot \nabla_{\varphi}^{\prime}(x y) .
$$

Clearly, (17) implies that (16) is equivalent to (15). The second assertion is proved in a similar way.

Note. Our proof followed the general pattern indicated in [8].

In particular, if $(\mathrm{g}, R)$ is a Baxter Lie algebra and $\left(\mathfrak{D}, R_{\mathfrak{b}}\right)$ its square, 
we get the structure of a Poisson Lie group for both $G$ and $D=G \times G$. We shall refer to the Poisson structure on $G_{(-R, R)}$ as the Sklyanin bracket.

Let us recall now the basic definition of [8]. Suppose $H$ is a Poisson Lie group, $\mathfrak{h}$ its Lie algebra, $\mathfrak{h}^{*}$ its dual. For $\xi, \eta \in \mathfrak{h}^{*}$ choose $\varphi, \phi \in C^{\infty}(H)$ such that $\xi=d_{e} \varphi, \eta=d_{e} \psi$ and put

$$
[\xi, \eta]_{*}=d_{e}\{\varphi, \psi\} \text {. }
$$

Theorem 3. [8]. (i) Formula (18) defines the structure of a Lie algebra on $\mathfrak{h}^{*}$. (ii) The map $\mathfrak{h}_{\rightarrow} \wedge^{2} \mathfrak{h}$ dual to the Lie bracket $\wedge^{2} \mathfrak{h}^{*} \rightarrow \mathfrak{h}^{*}$ is a 1 -cocycle on $\mathfrak{h}$.

We shall say that $\left(\mathfrak{h}, \mathfrak{h}^{*}\right)$ is a Lie bialgebra if there is a Lie bracket on $\mathfrak{h}^{*}$ satisfying condition (ii) above and refer to $\left(\mathfrak{h}, \mathfrak{h}^{*}\right)$ as the tangent Lie bialgebra of $H$.

Example. Let $(\mathfrak{g}, R)$ be a Baxter Lie algebra. The Lie bracket on $\mathfrak{g}^{*}$ defined by the Sklyanin bracket on $G$ coincides with (2) under the natural identification $\mathfrak{g}^{*} \simeq \mathfrak{g}$ induced by the inner product on $\mathfrak{g}$. Thus $\left(\mathfrak{g}, \mathfrak{g}_{R}\right)$ is an example of a Lie bialgebra.

Theorem 4. [8]. The structure of a Poisson Lie group is defined uniquely by its tangent Lie bialgebra.

As a corollary we can state the following result.

Proposition 2. Let $G$ be a Poisson Lie group. Its subgroup $H$ is a Poisson Lie subgroup if and only if $\mathfrak{h}^{\perp} \subset \mathrm{g}^{*}$ is an ideal. In that case the tangent Lie bialgebra of $H$ is $\left(\mathfrak{h}, \mathfrak{g}^{*} / \mathfrak{h}^{\perp}\right)$.

Specifically, let $\left(\mathfrak{D}, R_{\triangleright}\right)$ be the square of a Baxter Lie algebra $(g, R), D=G \times G$. The tangent Lie bialgebra of $D_{\left(R_{b^{\prime}}, R_{\mathrm{b}}\right)}$ coincides with $\left(\mathfrak{D}, \widehat{D}_{R_{d}}\right)$ under the isomorphism $\mathfrak{D}^{*} \simeq \mathscr{D}$ induced by the inner product. Clearly, $\mathfrak{D}_{R_{\mathrm{b}}}={ }^{\delta} \mathrm{g} \ominus \mathfrak{g}_{R}$ (i.e. it is a direct sum of ${ }^{\delta} \mathrm{g}$ and the opposite of $\mathrm{g}_{R}$ ). So we get

Proposition 3. Both ${ }^{\delta} G$ and $G_{R} \subset D$ are Poisson Lie subgroups. Their tangent Lie bialgebras are $\left(\mathfrak{g}, \mathfrak{g}_{R}\right)$ and $\left(\mathfrak{g}_{R}, \mathfrak{g}\right)$ respectively. 
In a sence, the Poisson structures on ${ }^{\delta} G$ and $G_{R}$ are dual to each other, the roles of $\mathrm{g}$ and $\mathfrak{g}_{R}$ being interchanged in their definition.

Note. In typical applications $\mathfrak{g}$ is a simple Lie algebra. Hence, $G_{R}$ does not contain any nontrivial Poisson subgroups.

Proposition 4. The Poisson structure on $G$ induced via the embedding $G \subset{ }^{\delta} G \subset D$ coincides up to a sign with the one defined by means of the original $\mathrm{r}$-matrix $R \in$ End $\mathrm{g}$.

This is again a direct corollary of Theorem 4 .

We shall give a more direct description of the Poisson structure on $G_{R}$ in the next section.

\section{§3. Classical r-Matrices and Lax Equations}

We shall now indicate how the $r$-matrix formalism is used to produce Lax equations on Lie groups. We start with the most simple theorem of this kind.

Let $G$ be a Lie group, $\mathfrak{g}$ its Lie algebra, $R \in$ End $\mathfrak{g}$. Suppose $(\mathfrak{g}, R)$ is a Baxter Lie algebra. We equip $G$ with the Sklyanin bracket defined by $R$. Let $I(G)$ be the space of central functions on $G$. Denote by $\lambda_{L}, \rho_{L}$ the differentials of left and right translations by an element $L \in G$.

Theorem 5. (i) Functions $\varphi \in I(G)$ commute with each other with respect to the Sklyanin bracket on $G$.

(ii) The equation of motion defined by a Hamiltonian $\varphi \in I(G)$ with respect to the Sklyanin bracket is given by

$$
\frac{d L}{d t}=\lambda_{L} M-\rho_{L} M, \quad M=\frac{1}{2} R\left(\nabla_{\varphi}\right) .
$$

For matrix groups (19) is a Lax equation,

$$
\frac{d L}{d t}=[L, M] \text {. }
$$

(iii) Let $g_{+}(t), g_{-}(t)$ be the solutions of the factorization problem (6) with the left hand side 


$$
g(t)=\exp t \nabla_{\varphi}\left(L_{0}\right) \text {. }
$$

The integral curve of equation (19) starting at $L_{0} \in G$ is given by

$$
L(t)=g_{ \pm}(t)^{-1} L_{0} g_{ \pm}(t) .
$$

It is not difficult to check (21) by a direct computation. However, a geometric proof which we are going to outline is more instructive since it reveals the mutial relations of various Poisson structures involved. The same considerations will also be of importance when we turn to the study of dressing transformations.

The next assertions are quite general and provide a basis for applying the reduction technique to the problems we are concerned with.

Let $G$ be a connected Poisson Lie group, $\left(g, g^{*}\right)$ its tangent Lie bialgebra. Suppose there is an action of $G$ on a smooth Poisson manifold $M$. For $\varphi \in C^{\infty}(M)$ let $\xi_{\varphi}(x)=\left.d_{g} \varphi(g \circ x)\right|_{g=e}, \xi_{\varphi} \in g^{*}$. Let $\hat{X}$ be the vector field on $M$ defined by an element $X \in \mathrm{g}$.

Proposition 5. The action $G \times M \rightarrow M$ is a Poisson action if and only if

$$
\hat{X}\{\varphi, \phi\}-\{\hat{X} \varphi, \phi\}-\{\varphi, \hat{X} \phi\}=\left\langle\left[\xi_{\varphi}, \xi_{\psi}\right]_{*}, X\right\rangle
$$

This statement is a direct consequence of the definitions.

Theorem 6. Let $G \times M \rightarrow M$ be a Poisson group action. Let $H \subset G$ be a connected Lie subgroup. Assume that $\mathfrak{h}^{\perp} \subset \mathrm{g}^{*}$ is a Lie subalgebra. Then the algebra $C^{H}$ of $H$-invariant functions is a lie subalgebra in $C^{\infty}(M)$.

Proof. Let $\varphi, \phi \in C^{H}$. Then $\hat{X} \varphi=\hat{X} \psi=0$ for $X \in \mathfrak{h}$ and $\xi_{\varphi}, \xi_{\psi} \in \mathfrak{h}^{\perp}$. From (22) we get immediately

$$
\hat{X}\{\varphi, \phi\}=\left\langle\left[\xi_{\varphi}, \xi_{\psi}\right]_{*}, X\right\rangle=0, X \in \mathfrak{h},
$$

whence $\{\varphi, \phi\} \in C^{H}$.

Corollary. Assume that the quotient space $H \backslash M$ is a smooth manifold. There is a unique Poisson structure on $H \backslash M$ such that the natural projection $\pi: M \rightarrow H \backslash M$ is a Poisson mapping。

Note that condition on $H$ in Theorem 6 is less restrictive than in Proposition 2. 
We shall refer to $\pi$ as the Poisson reduction map.

Note. The reduction technique (cf. [9]) is usually applied to Hamiltonian group actions only. For such actions the space of invariants is obviously a Lie subalgebra in $C^{\infty}$. However, the converse is not true, and the reduction technique can be easily extended to a more general setting. The necessary tools are provided by the notion of dual Poisson mappings explained in [9] (and going back to Lie). In the present context this technique was first applied by V. Drinfel'd to describe symplectic leaves of Poisson Lie groups (cf. theorem 7 below). Further applications were found by the author.

It is frequently useful to realize Poisson structures as quotients of a symplectic structure. An important tool for that is provided by squaring the group $G$.

Proposition 6. The Poisson structure on $D_{\left(R_{\mathfrak{b}}, R_{\mathfrak{b}}\right)}$ is nondegenerate.

Basically this observation (due to Drinfel'd) motivated the definition of the Poisson bracket on $D_{\left(R_{\mathrm{b}}, R_{\mathrm{b}}\right)}$. We now use it to describe a new Poisson structure on $G$.

Proposition 7. (i) The natural action of the diagonal subgroup ${ }^{8} G \subset D$ on $D_{\left(R_{\mathrm{b}}, R_{\mathrm{b}}\right)}$ is a Poisson action. (ii) Canonical projections $\pi^{\prime}: D \rightarrow^{\delta} G \backslash D$, $\pi: D \rightarrow D /{ }^{\circ} G$ are dual to each other in the sence of [9].

Proof. (i) This is a special case of Theorem 2. (ii) By definition, this means that left- and right ${ }^{\delta} G$-invariant functions on $D$ centralize each other with respect to the Poisson bracket on $D_{\left(R_{\mathrm{b}}, R_{\mathrm{b}}\right)}$. Suppose $\varphi \in C^{\infty}(D)$ is left- ${ }^{\delta} G$-invariant, $\phi \in C^{\infty}(D)$ is right $-{ }^{\delta} G$-invariant. Then $\nabla_{\varphi}, \nabla_{\psi}^{\prime} \in\left({ }^{\delta} \mathfrak{g}\right)^{\perp}={ }^{\delta} \mathfrak{g}$ and hence

$$
2\{\varphi, \phi\}_{\left(R_{\mathrm{b}}, R_{\mathrm{b}}\right)}=\left\langle R_{\mathrm{b}}\left(\nabla_{\varphi}\right), \nabla_{\psi}\right\rangle-\left\langle\nabla_{\varphi}^{\prime}, R_{\mathrm{b}}\left(\nabla_{\psi}^{\prime}\right)\right\rangle=0 .
$$

The natural model for the quotient spaces $D /{ }^{\delta} G,{ }^{\delta} G \backslash D$ is $G$ itself. Projections $\pi, \pi^{\prime}$ are given by

$$
\pi:(x, y) \mapsto x y^{-1}, \quad \pi^{\prime}:(x, y) \mapsto y^{-1} x .
$$


Proposition 8. Equip $G$ with the quotient Poisson structure. Its symplectic leaves coincide with the conjugacy classes in $G$.

Proof. According to a general theorem [9], symplectic leaves are

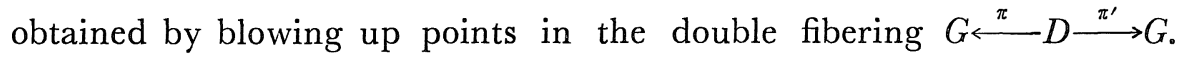
From (23) we get

$$
\pi^{\prime}\left(\pi^{-1}(g)\right)=\left\{h g h^{-1} ; h \in G\right\} .
$$

Corollary. Casimir functions of the quotient Poisson structure on $G$ are precisely central functions on G. (See [9] for the definition of Casimir functions.)

An explicit formula for the quotient Poisson bracket is given by

$$
\begin{aligned}
2\{\varphi, \phi\}= & \left(R\left(\nabla_{\varphi}^{\prime}\right), \nabla_{\psi}\right)+\left(R\left(\nabla_{\varphi}\right), \nabla_{\psi}^{\prime}\right) \\
& -\left(R\left(\nabla_{\varphi}\right), \nabla_{\psi}\right)-\left(R\left(\nabla_{\varphi}^{\prime}\right), \nabla_{\psi}^{\prime}\right)+\left(\nabla_{\varphi}^{\prime}, \nabla_{\psi}\right)-\left(\nabla_{\varphi}, \nabla_{\psi}^{\prime}\right) .
\end{aligned}
$$

To clarify the meaning of this Poisson structure we notice that another model of the quotient space $D /{ }^{\circ} G$ is $G_{R}$. The two models are related by the canonical map $\sigma: G_{R} \rightarrow G: h \mapsto h_{+} h_{-}^{-1}$. The next observations are basically due to V. Drinfel'd.

Proposition 9. The quotient Poisson structure on $G_{R} \simeq D /{ }^{8} G$ equips it with the structure of a Poisson Lie group. Its tangent Lie bialgebra is $\left(\mathfrak{g}_{R}, \mathfrak{g}\right)$.

Hence injection $G_{R} \subset D$ and projection $D \rightarrow G_{R}$ give the same Poisson structures on $G_{R}$ (at least up to sign). The dual assertion is also true.

Proposition 10. The quotient Poisson structure on $G \simeq D / G_{R}$ coincides with the Sklyanin bracket on $G$.

Proposition 11. Canonical projections $G_{R} \backslash D \leftarrow D \rightarrow D / G_{R}$ are dual to each other.

As a corollary we get the following theorem, again due to Drinfel'd.

Theorem 7. Symplectic leaves of the Sklyanin bracket coincide with 
projections of double cosets $G_{R} x G_{R} / G_{R}$.

We shall give a more explicit description of these symplectic leaves in Section 6.

We now turn to the proof of Theorem 5 .

Proposition 12. Let $\pi: D \rightarrow G:(x, y) \mapsto x y^{-1}$ be the standard projection, $\varphi \in I(G), h_{\varphi}=\varphi \circ \pi$. The integral curves of the Hamiltonian $h_{\varphi}$ on $D_{\left(R_{\mathfrak{b}}, R_{\mathfrak{b}}\right)}$ are given by

$$
\left(x_{0} e^{t X^{\prime}}, y_{0} e^{t X^{\prime}}\right), \quad X^{\prime}=\nabla_{\varphi}^{\prime}\left(x_{0} y_{0}^{-1}\right)
$$

Proof. Projections of the integral curve onto the quotient spaces $D /{ }^{\delta} G,{ }^{\delta} G \backslash D$ remain fixed since the reduced Hamiltonians are Casimir functions of the quotient Poisson structure. Since $h_{\varphi}$ is both rightand left- ${ }^{\delta} G$-invariant we have $\nabla_{h_{\varphi}}, \nabla_{h_{\varphi}}^{\prime} \in{ }^{\delta} \mathfrak{g}$ and for any $\phi \in C^{\infty}(D)$

$$
\left\{h_{\varphi}, \phi\right\}=\left\langle\nabla_{h_{\varphi}}^{\prime}, \nabla_{\psi}^{\prime}\right\rangle \text {. }
$$

Obviously, $\nabla_{h_{\varphi}}^{\prime}=\left(X^{\prime}, X^{\prime}\right) \in \mathfrak{d}, X^{\prime}=\nabla_{\varphi}^{\prime}(\pi(x, y))$ and $X^{\prime}$ is time independent. Now (25) follows immediately.

Consider the action $G_{R} \times D \rightarrow D$ defined by

$$
h:(x, y) \mapsto\left(h_{+} x h_{-}^{-1}, h_{+} y h_{-}^{-1}\right) .
$$

Notice that the subgroup $(G, e) \subset D$ is a cross section of (26). Hence we get a canonical projection

$$
\rho: D \rightarrow G:(x, y) \mapsto y_{+}^{-1} x y_{-}, \quad y=y_{+} y_{-}^{-1},
$$

whose fibers coincide with $G_{R^{-}}$orbits in $D$.

Proposition 13. (i) Invariants of (26) form a Lie subalgebra in $C^{\infty}(D)$. (ii) The quotient space is canonically isomorphic to $G_{(-R R)}$.

We shall give the proof of a more general statement (Theorem 9 below) in the Appendix.

To finish the proof of Theorem 5 we observe that for $\varphi \in I(G)$ the Hamiltonian $h_{\varphi}=\varphi \circ \pi$ is invariant with respect to (26). The integral curves (25) proiect down to $G$ to give (21). Moreover, the reduced Hamiltonian is $\varphi$ since $h_{\varphi}=\varphi \circ \rho$. 


\section{\$4. Twisted Poisson Structures on the Square of $G$ and the General Reduction Theorem}

Integrable systems on a lattice give rize to generalized Lax equations of the form

$$
\frac{d L}{d t}=L A-B L
$$

Equations of this kind are covered by the general reduction theorem which we shall state in this section.

Applications to integrable systems on a lattice will be considered in Section 5.

We keep to the notation of previous sections. Let $\tau$ be an automorphism of the Baxter Lie algebra $(\mathfrak{g}, R)$ i. e. an orthogonal operator $\tau \in$ Aut $\mathrm{g}$ which commutes with $R$. It gives rize to an automorphism of $G$ which we shall denote by $g \mapsto^{\tau} g$ 。 Define twisted conjugation $G \times G \rightarrow G$ by

$$
g: h \mapsto g h^{\tau} g^{-1} .
$$

Let ${ }^{\tau} I(G)$ be the space of smooth functions invariant with respect to twisted conjugations.

Theorem 8. (i) Functions $\varphi \in^{\tau} I(G)$ are in involution with respect to the Sklyanin bracket on $G$. (ii) Equations of motion defined by Hamiltonians $\varphi \in^{\tau} I(G)$ are of the form (27) with $B=\frac{1}{2} R\left(\nabla_{\varphi}\right), A=\tau(B)$. (iii) Let $g_{+}(t), g_{-}(t)$ be the solutions to the factorization problem (6) with the left hand side given by

$$
g(t)=\exp t \nabla_{\varphi}\left(L_{0}\right) .
$$

The integral curves of equation (27) defined bv $\varphi \in^{\tau} I(G)$ are given by

$$
L(t)=g_{ \pm}(t)^{-1} L_{0}^{\tau} g_{ \pm}(t) .
$$

The proof of (30) follows the same lines as in Theorem 5. However, we shall now twist the Poisson structure on $D$. Extend $\tau$ to $\mathfrak{d}=\mathfrak{g} \oplus \mathfrak{g}$ by the formula

$$
\hat{\tau}(X, Y)=(X, \tau Y)
$$

and put

$$
{ }^{\tau} R_{\mathrm{b}}=\hat{\tau} \circ R_{\mathrm{b}} \circ \hat{\tau}^{-1} .
$$


We also put ${ }^{\tau} G={ }^{\hat{\tau}}\left({ }^{\delta} G\right)=\left\{\left(x,{ }^{\tau} x\right) ; x \in G\right\} \subset D$.

Proposition 13. (i) The natural action of ${ }^{\tau} G$ on $D_{\left({ }^{\tau} R_{\mathrm{b}}, R_{\mathrm{b}}\right)}$ by left translations is a Poisson action. (ii) The natural action of ${ }^{\delta} G$ on $D_{\left({ }^{\tau} R_{b}, R_{b}\right)}$ by right translations is a right Poisson action.

This is a corollary of Theorem 2, since ${ }^{\delta} G \subset D_{\left(R_{\mathrm{b}},-R_{\mathrm{b}}\right)}$ and ${ }^{\tau} G \subset D_{\left({ }^{\tau} R_{\mathrm{b}},-{ }^{\tau} R_{\mathrm{b}}\right)}$ are Poisson subgroups.

Proposition 14. Canonical projections $\pi: D_{\left({ }^{\tau} R_{b}, R_{b}\right)} \rightarrow D /{ }^{\delta} G, \pi^{\prime}: D_{\left({ }^{\tau} R_{b}, R_{b}\right)}$ $\rightarrow^{\tau} G \backslash D$ are dual to each other.

Both quotient spaces are naturally modelled on $G$. Projections $\pi, \pi^{\prime}$ are given by

$$
\pi:(x, y) \rightarrow x y^{-1}, \quad \pi^{\prime}:(x, y) \mapsto^{\tau^{-1}} y^{-1} x .
$$

Proposition 15. Symplectic leaves in the quotient Poisson manifold $D /{ }^{\delta} G$ are orbits of twisted conjugations.

Proof. To get the symplectic leaves it suffices to compute $\pi\left(\pi^{\prime-1}(x)\right)$. Clearly,

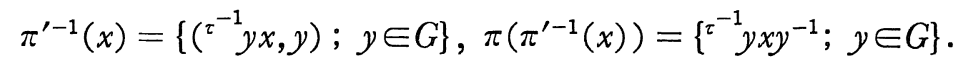

Corollary. Casimir functions of the quotient Poisson structure on $G$ are invariants of twisted conjugations (28).

We leave it to the reader to write down an explicit formula for the quotient Poisson structure on $G$.

Proposition 16. Let $\varphi \in{ }^{\tau} I(G), h_{\varphi}=\varphi \circ \pi$. Integral curves of the Hamiltonian $h_{\varphi}$ on $D_{\left({ }^{\tau} R_{\mathrm{b}}, R_{\mathrm{b}}\right)}$ are given by

$$
\left(x_{0} e^{t X^{\prime}}, y_{0} e^{t X^{\prime}}\right), \quad X^{\prime}=\nabla_{h_{\varphi}}^{\prime}\left(\pi\left(x_{0}, y_{0}\right)\right) .
$$

The proof is the same as in Proposition 12. It follows from the definition of $h_{\varphi}$ that $\nabla_{h_{\varphi}} \in{ }^{\tau} \mathfrak{g}, \nabla_{h_{\varphi}}^{\prime} \in{ }^{\delta} \mathrm{g}$ whence 


$$
\left\{h_{\varphi}, \phi\right\}=\left\langle\nabla_{h_{\varphi}}^{\prime}, \nabla_{\psi}^{\prime}\right\rangle
$$

for any $\phi \in C^{\infty}(D)$. Furthermore, $\nabla_{h_{\varphi}}^{\prime}(x, y)=\left(X^{\prime}, X^{\prime}\right)$, where $X^{\prime}=$ $\nabla_{\varphi}^{\prime}(\pi(x, y))$ is time independent.

Consider the action $G_{R} \times D \rightarrow D$ given by

$$
h:(x, y) \mapsto\left(h_{+} x h_{-}^{-1},{ }^{\tau} h_{+} y h_{-}^{-1}\right) .
$$

Theorem 9. (i) Invariants of (35) form a Lie subalgebra in $C^{\infty}\left(D_{\left({ }^{\tau} R_{\mathrm{b}}, R_{\mathrm{b}}\right)}\right)$ (ii) The quotient Poisson structure on $D / G_{R} \simeq G$ coincides with the Sklyanin bracket.

We shall give the proof in the Appendix. The first assertion is proved using Theorem 6. The calculation of the quotient Poisson structure is straightforward and includes some remarkable cancellations. This suggests that there should exist a more geometrical proof, but I did not found one as yet.

Note that the subgroup $(G, e) \subset D$ is again a cross section of (35). The canonical projection $\rho: D \rightarrow G$ is now given by

$$
\rho:(x, y) \mapsto^{\tau^{-1}} y_{+}^{-1} x y_{-}, \quad y=y_{+} y_{-}^{-1} .
$$

Again $h_{\varphi}=\varphi \circ \rho$, so (30) follows directly from (34), (36).

\section{§5. Difference Lax Equations}

An important application of Theorem 8 is described as follows. Given a Baxter Lie algebra $(\mathfrak{g}, R)$ and a corresponding Poisson Lie group $G$, put $\mathbb{G}=G^{N}, \mathscr{G}=\bigoplus^{N} \mathrm{~g}$. We shall think of elements $g \in \mathbb{G}$ as of functions mapping $\mathbb{Z} / N \mathbb{Z}$ into $G$. We equip $\mathscr{G}$ with the natural scalar product

$$
(X, Y)=\sum_{n}\left(X_{n}, Y_{n}\right)
$$

and extend $R \in$ End $g$ to $\mathscr{G}$ by $(R X)_{n}=R\left(X_{n}\right)$. This makes $(\mathscr{G}, R)$ a Baxter Lie algebra. Equip $G$ with the product Poisson structure. Clearly, $\boldsymbol{G}$ is a Poisson Lie group and its tangent Lie bialgebra is $\left(\mathscr{G}, \mathscr{G}_{R}\right)$. We shall denote elements of $G$ by $L=\left(L_{1}, \ldots, L_{N}\right)$. Define mappings $\phi_{m}, T: G \rightarrow G$ by

$$
\phi_{m}(L)=\widetilde{\Pi} L_{1 \leq k<m}, \quad T(L)=\widehat{\Pi} L_{1 \leq k \leq N} .
$$

The functions $\phi_{m}$ satisfy the linear difference system 


$$
\phi_{m+1}=\phi_{m} L_{m}, \quad m=1, \ldots, N,
$$

$T(L)$ is its monodromy matrix. Note that $T: G \rightarrow G$ is a Poisson map. This property had originally served as a motivation for the definition of the Sklyanin brackets. Subsequently it was formalized by $V$. Drinfel'd in his theory of Poisson groups [8].

Let $\tau \in$ Aut $\mathscr{G}$ be the cyclic permutation

$$
\tau:\left(X_{1}, \ldots, X_{N}\right) \mapsto\left(X_{2}, X_{3}, \ldots, X_{N}, X_{1}\right) .
$$

Clearly, the twisted coniugation $L \mapsto g L^{\tau} g^{-1}$ coincides with the gauge transformation for the linear system (39) induced by the right translation $\phi_{m} \mapsto \phi_{m} g_{m}^{-1}$ in its solution space. Obviously, the operator (40) is orthogonal and commutes with $R$. So we can apply Theorem 8 to our particular situation.

The gauge transformations orbits in $G$ can be easily classified.

Theorem 10 ("Floquet"). (i) Two elements $L, L^{\prime} \in G$ lie on the same gauge orbit in $G$ if and only if their monodromy matrices $T(L), T\left(L^{\prime}\right)$ are conjugate in $G$. (ii) The algebra ${ }^{\tau} I(G)$ is generated by the functions $h_{\varphi}: L \mapsto \varphi(T(L)), \varphi \in I(G)$.

As a corollary of Theorem 8 we get

Theorem $\mathbb{1}$. (i) Functions $h_{\varphi}, \varphi \in I(G)$, are in involution with respect to the Sklyanin bracket on $\mathbb{G}$. (ii) The Hamiltonian equation of motion with the Hamiltonian $h_{\varphi}$ is given by

$$
\begin{aligned}
& \frac{d L_{m}}{d t}=L_{m} M_{m+1}-M_{m} L_{m}, \\
& M_{m}=\frac{1}{2} R\left(\psi_{m}^{-1} \nabla_{\varphi}(T(L)) \phi_{m}\right) .
\end{aligned}
$$

(iii) Let $\left(g_{m}\right)_{ \pm}(t)$ be the solution to the factorization problem (6) with the left hand side given by

$$
g_{m}(t)=\phi_{m}^{0-1} \exp t \nabla_{\varphi}\left(T\left(L^{0}\right)\right) \psi_{m}^{0}, \phi_{m}^{0}=\psi_{m}\left(L^{0}\right) .
$$

The integral curve of (41) with the origin at $L^{0}=\left(L_{1}^{0}, \ldots, L_{N}^{0}\right)$ is given by

$$
L_{m}(t)=\left(g_{m}\right)_{ \pm}(t)^{-1} L_{m}^{0}\left(g_{m+1}\right)_{ \pm}(t)
$$


(For simplicity we assumed in (41) that $\mathbb{G}$ is a matrix group. The generalization is of course straightforward).

\section{\$6. Dressing Transformations}

We now turn to the definition of dressing transformations on the group $G_{0}$. Consider again the linear difference system (39). Let $\phi_{k}$ be its solution given by (38) i. e. satisfying the normalization condition $\phi_{1}=1$. (Note that arbitrary solutions of (39) may be obtained from (38) by a left translation $\left.\phi_{k} \mapsto h \psi_{k}, h \in G\right)$. The dressing action $\mathbb{G} \times G_{R} \rightarrow \mathbb{G}$ is defined by

$$
\left(L^{g}\right)_{n}=\left(\phi_{n}^{-1} \sigma(g) \psi_{n}\right)_{+}^{-1} L_{n}\left(\psi_{n+1}^{-1} \sigma(g) \phi_{n+1}\right)_{+}
$$

where $\sigma: G_{R} \rightarrow G: g \mapsto g_{+} g_{-}^{-1}$ is the canonical embedding.

Theorem 12. (i) Formula (43) defines a right Poisson action of $G_{R}$ on $\mathbb{G}$. (ii) This action preserves symplectic leaves in $\mathbb{G}$.

The proof will require some preparations. Let us notice first of all that in terms of the wave functions $\phi_{n}$ (i. e. solutions of (39)) (43) takes the form

$$
\psi_{n}{ }^{g}=g_{+}{ }^{-1} \psi_{n}\left(\psi_{n}^{-1} \sigma(g) \psi_{n}\right)_{+}, \quad g \in G_{R}
$$

The standard definition used in [1], [4] differs by the normalization factor $g_{+}$:

$$
\psi_{n}{ }^{g}=\phi_{n}\left(\phi_{n}^{-1} g \psi_{n}\right)_{+}, \quad g \in G .
$$

Note that the action (44) preserves the normalization condition $\phi_{1}=1$. We shall see readily that the normalization factor $g_{+}^{-1}$ in (44) affects the composition law of dressing transformations.

\section{Proposition 17. Formula}

$$
x^{g}=g_{+}^{-1} x\left(x^{-1} \sigma(g) x\right)_{+}
$$

defines a right group action $G \times G_{R} \rightarrow G$.

Proof. We shall point out a natural geometric interpretation of (46). Let $D=G \times G$ be the square of $G$. The quotient space $D / G_{R}$ is naturally modelled on the subgroup ${ }^{\delta} G \subset D$. Canonical projection $\rho$ : 
$D \rightarrow G$ is given by

$$
\rho:(x, y) \mapsto x\left(x^{-1} y\right)_{+} \cdot
$$

Now it is easy to check that natural action

$$
D \times G_{R} \rightarrow D:(x, y) g=\left(g_{+}{ }^{-1} x, g_{-}{ }^{-1} y\right)
$$

projects down to (46) under the map (47). In passing we note an identity

$$
g_{+}^{-1} x\left(x^{-1} \sigma(g) x\right)_{+}=g_{-}^{-1} x\left(x^{-1} \sigma(g) x\right)_{-} \cdot
$$

It is worth pointing out that the standard definition (45) corresponds to the action of the subgroup $(G, e) \subset D$ on $D / G_{R}$. We have preferred the definition (44) for two main reasons: first, $(G, e) \subset D$ is not a Poisson subgroup. Second, the action (45) does not preserve normalization of the wave functions and so does not lead to an action on the phase space. On the contrary, the Poisson properties of (44), (46) are quite nice. It goes without saying that all formulae of [1], [3], [4] relating dressing transformations to quantum field theory remain valid for the action (44) as well.

Theorem 13. (i) The dressing action (46) is a Poisson action. (ii) The symplectic leaf in $G$ containing a point $x$ is the orbit of $x$ under the dressing transformations (46).

Proof. (i) The natural action $D_{\left(R_{\mathrm{b}}, R_{\mathrm{b}}\right)} \times G_{R} \rightarrow D_{\left(R_{\mathrm{b}}, R_{\mathrm{b}}\right)}$ is a Poisson action which commutes with the projection $\rho: D \rightarrow G$. So it follows by standard machinery that the induced action on the quotient space is again a Poisson action. (ii) According to Proposition 11 the description of symplectic leaves in $G$ is related to the dual pair $D / G_{R}$ $\stackrel{\rho}{\longleftarrow} D_{\left(R_{\mathrm{b}}, R_{\mathrm{b}}\right)} \stackrel{\rho^{\prime}}{\longrightarrow} G_{R} \backslash D$. Both quotient spaces are naturally modelled on ${ }^{\delta} G \subset D$. The symplectic leaf containing $x \in G$ coincides with $\rho\left(\rho^{\prime-1}(x)\right)$. Clearly, $\rho^{\prime-1}(x)=\left\{\left(h_{+} x, h_{-} x\right) ; h \in G_{R}\right\}$. Our assertion now follows.

We shall now analyze the Poisson properties of the dressing action (43). Formula (44) is somewhat easier to deal with. However, first the Poisson structure on the space of solutions of difference system (39) should be studied.

Denote by $V$ the space of solutions to system (39) for arbitrary 
$L \in \boldsymbol{G}$ satisfying the normalization condition $\phi_{1}(L)=1$. We fix the Poisson structure on $V$ by demanding that

$$
\psi:\left.L \mapsto \phi_{n}(L)\right|_{n=2} ^{N+1}: G \rightarrow V
$$

be a Poisson mapping. Clearly, $\psi$ is a Poisson isomorphism. We shall need an explicit formula for the Poisson structure on $V$. It suffices to compute the Poisson brackets of "cylindrical" function on $V$ depending on the value of $\phi$ at one particular site $n$ each. We shall denote such functions $\varphi_{n}(\psi)=\varphi_{n}\left(\psi_{n}\right)$. Denote by $\nabla_{\varphi_{n}}, \nabla_{\varphi_{n}}^{\prime}$ the left and right gradient of $\varphi_{n}$ (regarded as a function of one variable $\phi_{n} \in G$ ).

Proposition 18. The Poisson bracket of functions $\varphi_{n}, \varphi_{m}$ on $V$ is given by

$$
2\left\{\varphi_{n}, \varphi_{m}\right\}_{V}(\psi)=\left(R\left(\nabla_{\varphi_{n}}^{\prime}\right), \phi_{n}^{-1} \nabla_{\varphi_{m}} \phi_{n}\right)-\left(R\left(\nabla_{\varphi_{n}}\right), \nabla_{\varphi_{m}}\right), \quad n \leq m .
$$

The proof is standard; we shall give it in the Appendix.

We have seen already that squaring the group $G$ is crucial for the study of Poisson structures on $G$ itself. So a natural way to study the Poisson bracket (48) is to realize it as a quotient Poisson structure by squaring the space $V$. Let $W=D \times \ldots \times D$. Elements $w \in W$ are sequences $\left(w_{n}\right)_{n=2}^{N+1}$. We define the Poisson bracket of cylindrical functions $\varphi_{n}, \varphi_{m}$ on $W$ by

$$
2\left\{\varphi_{n}, \varphi_{m}\right\}(w)=-\left\langle R_{\triangleright}\left(\nabla_{\varphi_{n}}^{\prime}\right), w_{n}^{-1} \nabla_{\varphi_{m}} w_{n}\right\rangle+\left\langle R_{\triangleright}\left(\nabla_{\varphi_{n}}\right), \nabla_{\varphi_{m}}\right\rangle, n \leq m .
$$

A comparison with (48) immediately shows that the definition is correct.

Proposition 19. Diagonal action $D \times W \rightarrow W$ by left translations is a Poisson action.

Proof. Rewrite (49) in the following form

$$
2\left\{\varphi_{n}, \varphi_{m}\right\}=\left\langle\eta_{n}\left(\nabla_{\varphi_{n}}\right), \nabla_{\varphi_{m}}\right\rangle
$$

where

$$
\eta_{n}(w)=R_{b}-\operatorname{Ad} w_{n} \circ R_{b} \circ \operatorname{Ad} w_{n}^{-1} .
$$

One checks immediately that

$$
\eta_{n}(h w)=\operatorname{Ad} h \circ \eta_{n}(w) \circ \operatorname{Ad} h^{-1}+R_{\mathrm{b}}-\operatorname{Ad} h \circ R_{\mathrm{o}} \circ \operatorname{Ad} h^{-1} .
$$


Since $\nabla_{\varphi_{k}}(h w)=\operatorname{Ad} h \cdot\left(\nabla_{\lambda_{h} \varphi_{k}}(w)\right)$, our assertion now follows from the definition of Poisson actions.

Define an action $W \times G_{R}^{N} \rightarrow W$ by right translations

$$
(w g)_{n}=w_{n} g_{n}, \quad g=\left(g_{n}\right)_{n=2}^{N+1} \in G_{R}^{N}
$$

Proposition 20. Right-G $G_{R}^{N}$-invariant functions on $W$ form a Lie subalgebra with respect to the Poisson bracket (49).

Proof. It suffices to prove that the Poisson bracket of two cylindrical $G_{R}^{N}$-invariant functions is again $G_{R}^{N}$-invariant. Note that $G_{R}^{N}$-invariance of $\varphi_{n}$ implies $R_{b}\left(\nabla_{\varphi_{n}}^{\prime}\right)=-\nabla_{\varphi_{n}}^{\prime}$. Hence the formula (49) simplifies to give

$$
\left\{\varphi_{n}, \varphi_{m}\right\}=\left\{\left(R_{\mathrm{b}}\right)_{+}\left(\nabla_{\varphi_{n}}\right), \nabla_{\psi_{m}}\right\rangle, \quad\left(R_{\mathrm{b}}\right)_{+}=\frac{1}{2}\left(R_{\mathrm{b}}+1\right) .
$$

Since left gradients are right-invariant our assertion now follows.

We embed $V \subset W$ via $\phi \mapsto\left(\phi_{n}, \phi_{n}\right)_{n=2}^{N+1}$. The quotient space $W / G_{R}^{N}$ is naturally modelled on $V$.

Proposition 21. The quotient Poisson structure on $W / G_{R}^{N} \simeq V$ coincides with (48).

The proof is again based on a straightforward computation. We give it in the Appendix.

We are able now to finish the proof of Theorem 12. Just observe that diagonal action $G_{R} \times W \rightarrow W$ projects down to the dressing action (44) on $V$ (this is basically the contents of Proposition 17). Since $D \times W / G_{R}^{N} \rightarrow W / G_{R}^{N}$ is a Poisson action and $G_{R} \subset D$ is a Poisson subgroup, our main assertion follows.

We conclude our paper with a few remarks on the continuous case. The definition of dressing transformations in the continuous case is given as follows. Let $\mathscr{G}=C^{\infty}(\mathbb{R} ; \mathfrak{g})$ be the current algebra associated with a Baxter Lie algebra $(\mathfrak{g}, R)$. We extend $R$ to $\mathscr{G}$ by $R X(x)=R(X(x))$ and define scalar product on $\mathscr{G}$ in the ordinary way 


$$
(X, Y)=\int d x(X(x), Y(x))
$$

The Poisson structure on $\mathscr{G}_{R}$ is the ordinary Lie-Poisson bracket

$$
\left\{\varphi_{1}, \varphi_{2}\right\}(L)=\left(L,\left[\operatorname{grad} \varphi_{1}(L), \operatorname{grad} \varphi_{2}(L)\right]_{R}\right) .
$$

Let $\phi(x)$ be the fundamental solution of the linear differential equation

$$
\partial_{x} \psi=\phi L
$$

normalized by $\phi(0)=1$.

Put

$$
L^{g}=\left(\phi \sigma(g) \psi^{-1}\right)_{+}^{-1}\left(\partial_{x}+L\right)\left(\phi \sigma(g) \psi^{-1}\right)_{+} \cdot
$$

Theorem 14. Formula (54) defines a right Poisson action $\mathscr{G}_{R} \times G_{R} \rightarrow \mathscr{G}_{R}$.

The proof follows the same lines as before with minor changes, so we shall not dwell upon it.

\section{Appendix}

We shall give here the proofs of several statements omitted in the main text.

\section{Proof of Theorem 9 .}

Embed $G_{R}$ into $D_{\left({ }^{\tau} R_{\mathfrak{b}},-\tau_{\left.R_{\mathfrak{b}}\right)}\right.} \times D_{\left(-R_{\mathfrak{b}}, R_{\mathfrak{b}}\right)}$ via

$$
h \mapsto\left(\hat{\imath}\left({ }^{\delta} h_{+}\right),{ }^{\delta} h_{-}\right) .
$$

Lemma A. $\mathbb{1}_{\text {. Natural action of }} D_{\left(\tau_{R_{\mathfrak{b}}},-\tau_{R_{\mathfrak{b}}}\right)} \times D_{\left(-R_{\mathfrak{b}}, R_{\mathfrak{b}}\right)}$ on $D_{\left({ }^{\tau} R_{\mathfrak{b}}, R_{\mathfrak{b}}\right)}$ given by $\left(g_{1}, g_{2}\right): x \mapsto g_{1} x g_{2}^{-1}$ is a Poisson action.

This assertion follows in a routine way from Theorem 2.

Clearly, the tangent Lie bialgebra of $D \times D$ is $\left(\mathfrak{D} \mathfrak{D}_{,} \mathfrak{D}_{-} \tau_{R_{\triangleright}} \oplus \mathfrak{D}_{R_{\mathfrak{b}}}\right)$.

Lemma A.2. Embed $\mathfrak{g}_{R}$ into $\mathfrak{D} \oplus \mathfrak{D}$ via the differential of (A. 1). Then $\mathfrak{g}_{R}^{\perp}$ is a Lie subalgebra in $\mathfrak{d}_{-}=_{R_{\mathfrak{b}}} \oplus \mathfrak{d}_{R_{\mathrm{b}}}$. 
Proof. An element $\left(\left(X_{1}, X_{2}\right),\left(Y_{1}, Y_{2}\right)\right) \in \mathfrak{d} \oplus \mathfrak{d}$ annihilates $\mathfrak{g}_{R}$ if and only if

$$
R_{-}\left(X_{1}-\tau^{-1} X_{2}\right)+R_{+}\left(Y_{1}-Y_{2}\right)=0 .
$$

Equivalently,

$$
\begin{gathered}
\mathfrak{g}_{R}^{\perp}=\left\{\left((\xi, \tau \xi)+\left(\eta_{+}, \tau \eta_{-}\right),\left(\xi^{\prime}, \xi^{\prime}\right)+\left(\eta_{+}^{\prime}, \eta_{-}^{\prime}\right)\right) ;\right. \\
\left.\xi, \xi^{\prime} \in \mathfrak{g}, \quad \eta, \eta^{\prime} \in \mathfrak{g}_{R}, \quad \eta_{-}+\eta_{+}^{\prime}=0\right\} .
\end{gathered}
$$

Since ${ }^{\delta} \mathfrak{g}, \mathfrak{g}_{R} \subset \mathfrak{D}_{R_{\mathfrak{b}}},{ }^{\tau} \mathfrak{g},{ }^{\tau} \mathfrak{g}_{R} \subset \mathfrak{D}_{-} \tau_{R_{\mathrm{b}}}$ are Lie subalgebras (at least, up to an anti-automorphism) it suffices to check that

$$
R_{-} \eta_{1}=-R_{+} \eta_{1}^{\prime}, \quad R_{1} \eta_{2}=-R_{+} \eta_{2}^{\prime}
$$

implies

$$
R_{-}\left(\left[\eta_{1}, \eta_{2}\right]_{R}\right)=-R_{+}\left(-\left[\eta_{1}^{\prime}, \eta_{2}^{\prime}\right]_{R}\right) .
$$

Now, the Yang-Baxter identity implies

$$
R_{-}\left(\left[\eta_{1}, \eta_{2}\right]_{R}\right)=\left[R_{-} \eta_{1}, R_{-} \eta_{2}\right]=\left[R_{+} \eta_{1}^{\prime}, R_{+} \eta_{2}^{\prime}\right]=R_{+}\left(\left[\eta_{1}^{\prime}, \eta_{2}^{\prime}\right]_{R}\right) .
$$

Q. E. D.

The first assertion of Theorem 9 now follows from Theorem 6 . Recall that the canonical projection $\rho: D \rightarrow G$ is given by

$$
\rho:(x, y) \mapsto \tau^{-1} y_{+}^{-1} x y_{-} .
$$

For $\varphi, \phi \in C^{\infty}(G)$ put $H_{\varphi}=\varphi \circ \rho, H_{\psi}=\phi \circ \rho$. Put $X=\nabla_{\varphi}, X^{\prime}=\nabla_{\varphi}^{\prime}, \quad Y=\nabla_{\psi}$, $Y^{\prime}=\nabla_{\psi}^{\prime}$. It is easy to check that the gradients of $H_{\varphi}$ restricted to the surface $(G, e) \subset D$ are given by

$$
\text { (A. 3) } \quad \nabla_{H_{\varphi}}=\left(X, X_{+}^{\prime}-\tau X_{-}\right), \quad \nabla_{H_{\varphi}}^{\prime}=\left(X^{\prime}, X_{+}^{\prime}-\tau X_{-}\right) \text {. }
$$

Similar formulae hold for the gradients of $H_{\psi}$. Now,

$$
\begin{aligned}
& R_{b}\left(\nabla_{H_{\varphi}}^{\prime}\right)=\left(X^{\prime}, X^{\prime}-X_{-}^{\prime}+\tau X_{-}\right), \\
& { }^{\tau} R_{b}\left(\nabla_{H_{\varphi}}\right)=\left(2 \tau^{-1} X_{+}^{\prime}-X_{+}-X_{-}, X_{+}^{\prime}-\tau X_{-}\right) .
\end{aligned}
$$

Hence

$$
\begin{aligned}
\left.2\left\{H_{\varphi}, H_{\psi}\right\}\right|_{(G, e)}= & \left\langle\left(2 \tau^{-1} X_{+}^{\prime}-X_{+}-X_{-}, X_{+}^{\prime}-\tau X_{-}\right), \quad\left(Y, Y_{+}^{\prime}-\tau Y_{-}\right)\right\rangle \\
& +\left\langle\left(X^{\prime}, X^{\prime}-X_{-}^{\prime}+\tau X_{-}\right),\left(Y^{\prime}, Y_{+}^{\prime}-\tau Y_{-}\right)\right\rangle \\
= & 2\left(\tau^{-1} X_{+}^{\prime}, Y\right)-\left(X_{+}, Y\right)-\left(X_{-}, Y\right)-\left(X_{+}^{\prime}, Y_{+}^{\prime}\right) \\
& +\left(\tau X_{-}, Y_{+}^{\prime}\right)-\left(\tau X_{-}, \tau Y_{-}\right)+\left(X_{+}^{\prime}, \tau Y_{-}\right)+\left(X^{\prime}, Y^{\prime}\right) \\
& -\left(X^{\prime}, Y_{+}^{\prime}\right)-\left(\tau X_{-}, Y_{+}^{\prime}\right)+\left(X_{-}^{\prime}, Y_{+}^{\prime}\right)+\left(X^{\prime}, \tau Y_{-}\right)
\end{aligned}
$$




$$
\begin{aligned}
& +\left(X_{-}, \tau Y_{-}\right)-\left(X_{-}^{\prime}, \tau Y_{-}\right) \\
= & \left(X_{+}^{\prime}, Y^{\prime}\right)-\left(X^{\prime}, Y_{+}^{\prime}\right)-\left(X_{+}, Y\right)+\left(X, Y_{+}\right)=2\{\varphi, \phi\}_{G} .
\end{aligned}
$$

\section{Proof of Proposition 18.}

Linear difference system (39) implies, by the ordinary variational method, that

$$
\begin{aligned}
& \nabla_{\varphi_{n} \circ \psi}(L)=\left(\psi_{k}^{-1} \nabla_{\varphi_{n}} \phi_{k}\right)_{k=1}^{n-1}, \\
& \nabla_{\varphi_{n} \circ \psi}^{\prime}(L)=\left(\psi_{k+1}^{-1} \nabla_{\varphi_{n}} \phi_{k+1}\right)_{k=1}^{n-1}
\end{aligned}
$$

Hence

$$
\text { (A. 6) } \begin{aligned}
2\left\{\varphi_{n} \circ \phi, \varphi_{m} \circ \psi\right\}(L)= & \sum_{k=1}^{n-1}\left(R\left(\phi_{k+1}^{-1} \nabla_{\varphi_{n}} \phi_{k+1}\right), \phi_{k+1}^{-1} \nabla_{\varphi_{m}} \phi_{k+1}\right) \\
& -\sum_{k=1}^{n-1}\left(R\left(\psi_{k}^{-1} \nabla_{\varphi_{n}} \phi_{k}\right), \quad \phi_{k}^{-1} \nabla_{\varphi_{m}} \phi_{k}\right), \quad n \leq m .
\end{aligned}
$$

The right hand side of (A.6) is a total difference; after cancellations we get (48).

\section{Proof of Proposition 21.}

Let $\varphi_{n}, \varphi_{m}(n \leq m)$ be cylindrical function on $V$. Extend them to right $G_{R}^{N}$-invariant functions $\hat{\varphi}_{n}, \hat{\varphi}_{m}$ on $W$. Recall that we have denoted $\nabla_{\varphi_{n}}, \nabla_{\varphi_{n}}^{\prime}$ the gradients of $\varphi_{n}$ regarded as a function of one variable. Put $\nabla_{\varphi_{n}}=X_{n}, \nabla_{\varphi_{n}}^{\prime}=X_{n}^{\prime}$. One checks that

$$
\left.\nabla_{\hat{\varphi}_{n}}^{\prime}\right|_{V}=\left(\left(X_{n}^{\prime}\right)_{+},\left(X_{n}^{\prime}\right)_{-}\right) \in \mathcal{D}_{。}
$$

Hence

$$
\text { (A. 7) }\left.\quad \nabla_{\hat{\varphi}_{n}}\right|_{V}=\left(\psi_{n}\left(X_{n}^{\prime}\right)_{+} \psi_{n}^{-1}, \psi_{n}\left(X_{n}^{\prime}\right)_{-} \psi_{n}^{-1}\right) \text {. }
$$

Now, (51) and (A.7) imply

$$
\begin{aligned}
& \left\{\hat{\varphi}_{n}, \hat{\varphi}_{m}\right\}(\phi)=\left\langle P_{\delta_{\mathrm{g}}}\left(\nabla_{\hat{\varphi}_{n}}\right), \nabla_{\hat{\varphi}_{m}}\right\rangle \\
& =\left\langle\left(\left(\phi_{n}\left(X_{n}^{\prime}\right)_{-} \phi_{n}^{-1}\right)_{+}-\left(\phi_{n}\left(X_{n}\right)_{+} \psi_{n}^{-1}\right)_{-},\left(\phi_{n}\left(X_{n}^{\prime}\right)_{-} \phi_{n}^{-1}\right)_{+}\right.\right. \\
& \left.\left.-\left(\phi_{n}\left(X_{n}\right)_{+} \phi_{n}^{-1}\right)_{-}\right),\left(\phi_{m}\left(X_{m}^{\prime}\right)_{+} \phi_{m}^{-1}, \phi_{m}\left(X_{m}^{\prime}\right)_{-} \phi_{m}^{-1}\right)\right\rangle \\
& =\left(\left(\phi_{n}\left(X_{n}^{\prime}\right)_{-} \psi_{n}^{-1}\right)_{+}, \phi_{n}\left(X_{m}^{\prime}\right)_{+} \phi_{m}^{-1}\right)-\left(\left(\phi_{n}\left(X_{n}^{\prime}\right)_{+} \phi_{n}^{-1}\right)_{-}\right. \text {, } \\
& \left.\phi_{m}\left(X_{m}^{\prime}\right)_{+} \psi_{m}^{-1}\right)_{-}-\left(\left(\phi_{n}\left(X_{n}^{\prime}\right)_{-} \psi_{n}^{-1}\right)_{+}, \phi_{m}\left(X_{m}^{\prime}\right)_{-} \psi_{m}^{-1}\right) \\
& +\left(\left(\phi_{n}\left(X_{n}^{\prime}\right)_{+} \psi_{n}^{-1}\right)_{-}, \phi_{m}\left(X_{m}^{\prime}\right)_{-} \psi_{m}^{-1}\right) \\
& =\left(\left(\phi_{n}\left(X_{n}^{\prime}\right)_{-} \psi_{n}^{-1}\right)_{+}, \phi_{m} X_{m}^{\prime} \psi_{m}^{-1}\right)-\left(\left(\psi_{n}\left(X_{n}^{\prime}\right)_{+} \psi_{n}^{-1}\right)_{-}, \phi_{m} X_{m}^{\prime} \psi_{m}^{-1}\right)
\end{aligned}
$$




$$
\begin{aligned}
& =-\left(\phi_{n}\left(X_{n}^{\prime}\right)_{-} \psi_{n}^{-1},\left(X_{m}\right)_{-}\right)+\left(\psi_{n}\left(X_{n}^{\prime}\right)_{+} \psi_{n}^{-1},\left(X_{m}\right)_{+}\right) \\
& =\left(\psi_{n}\left(X_{n}^{\prime}\right)_{+} \psi_{n}^{-1}, X_{m}\right)+\left(\psi_{n}\left(X_{n}^{\prime}\right)_{+} \psi_{n}^{-1}-\psi_{n}\left(X_{n}^{\prime}\right)_{-} \psi_{n}^{-1},\left(X_{m}\right)_{-}\right) \\
& =\left(\left(X_{n}^{\prime}\right)_{+}, \phi_{n}^{-1} X_{m} \psi_{n}\right)-\left(\left(X_{n}\right)_{+}, X_{m}\right)
\end{aligned}
$$

\section{References}

[1] Date E., Jimbo M., Kashiwara M. and Miwa T., Transformation groups for soliton equation. Proc. Japan. Acad. 57A (1981). 3806-3818, Physica 4D (1982), 343-365; Publ. RIMS Kyoto Unvv., 18 (1982), 1077-1119.

[2] Zakharov V. E. and Shabat A. B., Integration of nonlinear equations by the inverse scattering method II, Funct. Anal. and its applications 13 (1979), 166-174.

[3] Segal G. and Wilson G., Loop groups and equations of KdV type, Publ. Math. I. H. E. S., 21 (1985), 1-64

[4] Wilson G., Habillage et fonctions $\tau$, C. R. Acad. Sci. Paris, 299 (1984), 587-590.

[5] Dolan L., Kac-Moody algebras and exact solvability in hadronic physics, Phys. Rep. 109 (1984), 1-94.

[6] Davies M. C. et al. Hidden symmetries as canonical transformations for chiral model, Phys. Lett., 119B (1982), 187-192.

[7] Semenov-Tian-Shansky M. A., What is the classical $r$-matrix, Funct. Anal. and its Applications, 17 (1983), 259-272.

[8] Drinfel'd V. G., Hamiltonian structures on Lie groups, Lie bialgebras and the geometric meaning of Yang-Baxter equations, DAN SSSR, 268, 285-287, in Russian.

[9] Weinstein A., Local structure of Poisson manifolds, J. Diff. Geom., 18 (1983), 523-558.

[10] Faddeev L. D., Integrable models in $1+1$ dimensional quantum field theory, Les Houches Lectures 1982.

[11] Gel'fand I. M. and Dorfman I. Ya., Hamiltonian operators and the classical YangBaxter equation, Funkz. analiz $i$ ego prilozh., 16 (1982), no. 4, 1-9, in Russian. 